\title{
HEYCITY: A SOCIAL-ORIENTED APPLICATION AND PLATFORM ON THE CLOUD
}

\section{Maximiliano Rasguido and Alex Villazón}

\begin{abstract}
Every city has problems related to infrastructure, services or security. Unfortunately, most cities lack of an on-line service or platform where citizens can complain about those problems. Typically there is only an email address or phone number where they can call, but only during working hours, which often results in frustration and long processing time until local authorities can solve those problems. In this article, we present HeyCity, a social-oriented application and platform that addresses this problem, by making the citizen an active part of the solution, and therefore increases responsibility. HeyCity provides a technological answer where users can report problems using their smartphone and collaborate with other citizens and local authorities to solve the problems. To be able to handle a large number of users distributed in different cities, HeyCity was deployed on the Cloud. We describe the design, development, deployment and execution of HeyCity on state-of-the-art Cloud services and tools, and we describe the technical choices that we made.
\end{abstract}

Keywords: Social-Oriented Application and Platform, Cloud Computing, PaaS, IaaS, DBaaS. 